Mechthild Albert

(Saarbrücken)

\title{
IMAGINIERTES AMERIKA, ERINNERTES SPANIEN, ERLEBTES ARGENTINIEN: RAMÓN GÓMEZ DE LA SERNA IN BUENOS AIRES
}

Nous n'avons pas été en Amérique, mais une fraternité fatale nous fait penser à ses paysages, parce que la nostalgie du crépuscule cherche les endroits d'élection: ceux où l'on naquit pour la seconde fois, ceux où un frère à nous a vu le jour, ceux par où passa notre père sans nous en avoir jamais rien dit. (PA, 314)

Mit diesen Worten beginnt Ramón Gómez de la Serna eine Serie von literarischen Skizzen, die in den Jahren 1922 und 1923 unter dem Titel »Paysages imaginaires d'Amérique« bzw. »Nouveaux paysages imaginaires d'Amérique«, übersetzt von Francis de Miomandre, in der Pariser Revue de l'Amérique Latine erscheinen. Ramón entwirft in diesen Prosagedichten knappe Szenen und Landschaftsbilder einer imaginären Neuen Welt - teils onirische "paysage[s] de l'inconscience«, teils klischeehafte Topoi oder preziöse greguerias. Amerika erweist sich hier einmal mehr als »endroit d'élection" der Avantgarde, die auf den fernen Kontinent ihre Sehnsucht nach dem Ursprünglichen, Primitiven, nach dem verlorenen Paradies projiziert. $^{2}$ Im Geiste eines solchen Exotismus greift Ramón auf mythische Vorstellungen aus der Zeit der Konquistadoren zurück. Er erwähnt Häuser, die im Winde schwanken, wundertätiges Heilwasser, Silberglöckchen, die in Bäumen Sphärenmusik von sich geben oder schwimmende Inseln:

Ce sont des îles qui échappent à l'explorateur et qui portent leur verdure exultante et leur virginité sur les mers d'Amérique, en lui envoyant le parfum de leurs grands basilics. (NPA, 198)

1 Siglen der zitierten Primärliteratur: PA $=$ Paysages imaginaires d'Amériques«, Revue de l'Amérique Latine I: 4 (1922), pp. 314-320; NPA = "Nouveaux paysages imaginaires d'Amérique«, Revue de l'Amérique Latine IV: 3 (1923), 193-199; EBA = Explicación de Buenos Aires (1948), Buenos Aires: La Flor, 1975; CMM = Cartas a mi mismo (1956), Madrid: Espasa-Calpe, 1962; NPV = Nuevas páginas de mi vida (1957), Madrid: Alianza, 1970. Die Stadttexte Ramóns liegen inzwischen vor als vol. XV der von Iona Zlotescu besorgten Gesamtausgabe: La ciudad. Madrid, Buenos Aires (1919-1956), Barcelona: Galaxia Gutenberg, 1999.

2 Cf. Michael Rössner (1988) 
Ähnliche Amerika-Topoi finden sich bereits einige Jahre zuvor in dem Porträt, das Ramón anlässlich des `Salón de los Íntegros〈 (1915) von Diego Rivera zeichnet. ${ }^{3}$ Poetologische Qualität gewinnt die avantgardistisch geprägte Lateinamerika-Exotik in Gestalt eines >Blauen Flusses‘, Gegenbild zur >Blauen Blume` der Romantik. Diesem Gewässer vertrauen die Dichter ihre misslungenen oder verheißungsvollen Werke an - in Gestalt von Papierschnipseln oder gefalteten Papierbooten:

Il y a un fleuve bleu, trouble à force d'être bleu, qui est celui qui emporte avec soi toutes les jeunesses, le fleuve où se soulagent tous les romantismes, le fleuve où les poètes jettent leurs poèmes, déchirés en petits morceaux ou pliés en forme de petits bâteaux. (NPA, 199)

Wie das Bild vom >Blauen Fluss` der jugendlichen Inspiration sind sämtliche Tableaux dieser »Paysages imaginaires « antimimetisch und fiktional zu verstehen; sie wollen keine Aussage über die Wirklichkeit treffen, sondern besitzen eindeutig Kunstcharakter:

Sur cette scène de paysages imaginaires il peut y avoir une erreur, mais il faut l'affronter. Pourquoi devrais-je nier l'évidence de ce que je n'ai pas vu? (PA, 319)

Ohne eine direkte eigene Anschauung des Fremden zu besitzen, gefällt sich Ramón in der Pose des voyageur immobile, der eine Forschungsreise in seine eigenen Phantasiewelten unternimmt, »dans cette Amérique que je parcours comme un explorateur [...] qui ne l'a jamais explorée« (NPA, 196). Immer wieder betont Ramón die Tatsache, dass es sich beim Betrachter bzw. Schöpfer dieser Bilder um einen Spanier handelt, der nie in Amerika gewesen ist - »qui n'a pas été en Amérique« (PA, 318) -, der mithin über eine rein euro-, ja hispanozentrische Perspektive verfügt, und unterstreicht damit den artifiziellen Charakter seiner Prosagedichte:

Je suis en Espagne - ces paysages sont toujours composés en prenant pour point de départ mon balcon qui est en Espagne - et je le [scil. le couchant américain] vois. (PA, 314)

Fünfundzwanzig Jahre nach Erscheinen dieser vignettenartigen Phantasiestücke, das heißt im Jahre 1948, haben sich die raum-zeitlichen Koordina-

3 In: Ramón en cuatro entregas, editado por Juan Manuel Bonet, Madrid: Museo Municipal, 1980, vol. II, p. 82. Cf. Mechthild Albert (1991: 150). 
ten in Ramóns Verhältnis zu Amerika verändert, ja umgekehrt. Denn in der Zwischenzeit hat er einen anderen >Balkon $<$ betreten, der ihm eine neue Sicht der Dinge eröffnet. In den Jahren 1931 und 1933 nämlich hatte Ramón anlässlich zweier Vortragsreisen nach Argentinien nicht nur einen Teil von Amerika, sondern auch seine spätere Frau, Luisa Sofovich, kennengelernt, mit der er bei Ausbruch des Spanischen Bürgerkriegs endgültig nach Buenos Aires übersiedelt. Was die Dimension der Zeit betrifft, so ist der historische Moment der Avantgarden inzwischen vorüber, und Ramón verwandelt sich zusehends in den anachronistischen »superviviente de una época « ${ }^{4}$. Im Windschatten der weltgeschichtlichen Katastrophen zählt sich der Emigrant zu den »supervivientes universales«, die bleiben, um den Nachgeborenen die , Welt von gestern zu dokumentieren. ${ }^{5}$ Auch die Raumerfahrung des einstigen Avantgardisten ist dezentriert, denn der Mittelpunkt des Ramónschen Universums, die tertulia des Café Pombo, hat sich aufgelöst. Geographisch befindet er sich an den Antipoden Spaniens, um die Welt nunmehr von Buenos Aires aus zu betrachten:

Internacional, con aires universales de hogar nuevo, del otro lado de los horizontes europeos, accedida por barcos, por aviones y por trenes panamericanos, Buenos Aires es el gran balcón de la perspectiva, el sitio desde el que se ven todos los panoramas. (EBA, 188)

Indem er den amerikanischen gegen den europäischen Standpunkt eintauscht, vertauschen sich die Kategorien des »lado de acá« und des »lado de allá«, um mit Julio Cortázar zu sprechen. Der Ortswechsel von Madrid nach Buenos Aires impliziert also einen Perspektivwechsel, verfügt doch Amerika über eine eigene Optik - »Unos lentes especiales, los lentes de América« (EBA, 189) -, die einen unbezweifelbaren Erkenntnisgewinn verspricht:

Nosotros ganamos la posición envidiable de estar en «otro mundo» y de poder orientar el telescopio desde ese otro mundo. Por eso no tiene perdón de Dios el

4 Cf. das Kapitel "Ya soy un superviviente« (NPV, 107-110), wo es heißt: »Ha aumentado nuestra edad sin darnos cuenta y nos vamos convirtiendo en supervivientes de una época« (NPV, 107). Der "cargo honorifico de ssuperviviente«« wird definiert als »la jubilación más allá de la jubilación« (NPV, 108).

5 Cf. NPV, 109: "Ya somos como esos anticuarios que, metidos en su tienda de antigüedades, convierten en más antiguas sus cosas con sólo estar sentados en su sillón frailero en medio de ellas. ¿Y si dado lo que se promete de genicidios y trituraciones, todos los lejanos a los campos de la guerra, adquiriremos el máximo título de ssupervivientes universales` y seremos los que quedemos para contar cómo era el mundo?« 
que aquí oblitera su visión con parcialidades sin grandeza, notándose entonces que perdura en sus ojos la oftalmía que trajo de su lugar de origen y que no se ha adaptado a esta grandeza de miras, a esta capacidad tranquila de ver a lo lejos que tiene el Balcón de Buenos Aires. (EBA, 189-190)

Bereits im Jahre 1926 hatte Ramón in einer emphatischen »Salutación « für die ihm gewidmete Sondernummer der Zeitschrift Martin Fierro geschrieben:

Siempre vivimos frente a un río de dos orillas. La de enfrente para toda nuestra vida, nuestro pensar y nuestro mirar es América. ${ }^{6}$

Nun ist er selbst am anderen Ufer angelangt, und sein Bild von Amerika wird sich zwangsläufig verändern: von der Imagination zur Realität, von den Mythen der Avantgarde zur Wahrnehmung der Wirklichkeit, von der unberührten Urlandschaft zur kosmopolitischen Metropole. Als literarisches Medium verwendet er den gleichen assoziativ-beschreibenden Diskurs, den man aus seinen Stadttexten ${ }^{7}$ zu Madrid wie El rastro (1910) kennt, in denen er ein Mosaik urbaner Kuriositäten versammelt. Auf ähnliche Weise entfaltet er in Explicación de Buenos Aires einen neokostumbristischen Bilderbogen, der ebenso wie seine Autobiographie Automoribundia im Jahr 1948 erscheint. Welche Absicht er als Spanier mit diesem Werk verfolgt, das einem spanischen Publikum zugedacht ist, erläutert er im Prolog:

He procurado dar a mis amigos y compatriotas una clave de BA para que se paseen por sus calles y conozcan sus matices como si hubiesen desembarcado en la gran ciudad, tan americana, tan madrileña y tan barcelonesa.

Mi apreciación de los detalles que explican la ciudad está hecha desde aquí y desde allí, en una perspectiva de tiempo y mar.

He querido ver las diferencias y la intimidad de la familiar y extraña ciudad, centro optimista del porvenir, consuelo de finales peregrinos. (EBA, 7)

Auf der Grundlage von Kriterien, die er aus beiden Welten bezieht - »desde aquí y desde allí« -, möchte Ramón aufschlussreiche »detalles« und »matices« referieren, welche die Eigenheiten der argentinischen Metropole in ihrer Fremdheit und Vertrautheit deutlich machen - »las diferencias y la intimidad de la familiar y extraña ciudad«. Im Folgenden wollen wir an-

6 Ramón en cuatro entregas, vol. IV, p. 16; cf. Mechthild Albert (1991: 148).

7 Zu dieser Diskursmodalität $\mathrm{cf}$. Andreas Mahler (1999: 11-36). 
hand der Explicación de Buenos Aires beobachten, inwieweit Ramón das Fremde in seiner Differenz darzustellen versucht, um dann aber immer stärker das Eigene, Vertraute, Spanische in Lateinamerika wiederzufinden. Die Befindlichkeit des Emigranten widerspiegelnd, entfaltet sich das Amerikabild im Spannungsfeld von Zukunft und Vergangenheit - »centro optimista del porvenir, consuelo de finales peregrinos «. Die gleiche Ambivalenz zwischen dem zukunftsorientierten Optimismus des einstigen Avantgardisten und der Spanien-Nostalgie des Emigranten konstatiert man in den Nuevas páginas de mi vida aus dem Jahre 1957, wo es in dem apologetischen Kapitel »Por qué estoy en América« heißt:

[...] aquí está el gran laboratorio anchuroso y con luces finales.

Mi deber de precursor, de inventor durante toda la vida de cosas nuevas e insólitas me ha dado derecho a alcanzar un sitio en América, el lugar nuevo donde, según Ortega, está «la juventud del mundo».

En verdad, éste es el escondrijo en que me he retirado de entrambos mundos y donde me mantengo «en estado de llegada», que ese es el único consejo que debe persistir en el emigrante artístico. (NPV, 172-173)

Hier findet sich ein letztes Echo des jugendlichen Impetus der Avantgarde, wie wir ihn aus der »Salutación« für Martín Fierro kennen (1926). ${ }^{8}$ Doch die avantgardistische Emphase hat sich verloren, stattdessen manifestiert sich der Wunsch nach Erkenntnis des Fremden im Vergleich beider Welten: »una gran afición a comprender el mundo y a ver los dos hemisferios reunidos« (EBA, 10). Die unmittelbare Anschauung der Neuen Welt führt zur Revision von Klischees - auch der eigenen. So dementiert Ramón implizit eben jenen paisaje imaginario, den er selbst Jahre zuvor entworfen hatte:

Ya lo sabéis, hombres de imaginación tropical que estáis lejos del trópico, aquí no hay marinas ni paisajes de estos en que los cactus echan por alto sus pies espinados; aquí se vive una ciudad utópica y europea que sorprende porque es otra cosa que la que se pensaba, y se desemboca en una Gran Vía sin preocupaciones, pero también con muchas camiserías. (EBA, 162)

Hier kündigt Ramón das avantgardistische wie triviale Exotismus-Paradigma der Neuen Welt auf. Als Strategie der Demythifizierung betont er die europäische Alltäglichkeit, ja Banalität der argentinischen Metropole. Dadurch macht er deren Wirklichkeit assimilierbar - weniger vielleicht für 
sein spanisches Publikum als vielmehr für ihn selbst, den Emigranten, der angestrengt darauf bedacht ist, der Fremde einen Anschein von Normalität zu verleihen. Daneben stürzt er einige argentinische Mythen wie den des Gaucho oder des Tango. Das diesbezügliche Kapitel überrascht durch seinen Mangel an Klischees. Ramón lässt dagegen viel Platz für aussagekräftige Originalzitate und betont seine frühe Initiation in den Geist des Tango durch das Gespräch mit Argentiniern in Paris ${ }^{9}$ und Madrid:

[...] me di cuenta de lo que significaba [el tango], del alma sudamericana, que de antiguo, mucho antes de venir, había absorbido en libros, revistas y conversaciones con los argentinos de Madrid y de París. (EBA, 27)

Prüfstein des Argentinien-Bildes ist zweifellos der Umgang mit der Figur des Gaucho ${ }^{10}$. Ramón scheint dabei zunächst dem Kitsch der Pampa-Ikone zu erliegen, deren Porträt er in kurioser Mixtur aus exotismo und casticismo mit orientalischen und kastilischen Reminiszenzen überfrachtet:

El gaucho es una figura noble de campesino que parece remontarse a los orígenes. - Con su sombrero blando, barbado, poncho - chilaba distinguida -, facón, pantalón bombacha, al que orla a veces una extraña prenda de ropa blanca escarolada, botas de montar y espuelas de estrella, es el hidalgo rústico del campo. (EBA, 139-140)

Dann bricht er jedoch mit dem Klischee, um die Unangemessenheit der Simili-Folklore bloßzustellen:

En las grandes avenidas de la ciudad más europea de América sólo se ve al gaucho cuando se quiere dar color local a alguna demonstración nacional, y lograr oír a unos buenos payadores es mucho más difícil que encontrar un buen «cantaor» de flamenco en la Puerta del Sol.

No se nos quiera ver entre barbudos de endrina barba montados a caballo con estribos de taza, ni establecidos entre la florida disputa, retruecada con aforismos y refranes aconsonantados en payadas de café pues eso sería inventar la «americanada» frente a la «españolada». (EBA, 142)

Jenseits der touristischen und exotischen Fremdwahrnehmung sucht Ramón das verborgene Innenleben der lateinamerikanischen Großstadt zu entdecken:

9 Cf. die einschlägigen Beiträge in dem Sammelband Paris et le phénomène des capitales littéraires (1984).

10 Cf. Donald S. Castro (1995). 
El novelista tiene aquí un gran mundo que descifrar con difícil cuidado, tomándose mucho tiempo, dando con la entraña popular que está debajo de su cosmopolitismo de brillantes tonos.

Yo procuro internarme en el secreto de la monumental vida - monumental por su calidad vital más que por sus monumentos - y dar al español lejano la síntesis rimbombante y escabrosa del variante y costoso Buenos Aires. (EBA, 152)

Wenn er behauptet, die Geheimnisse der Stadt erschließen zu wollen, suggeriert er ein hermeneutisches Interesse, das er letztlich nicht einlöst. Auch eine Synthese mag ihm angesichts der Vielfalt und Komplexität der argentinischen Wirklichkeit nicht gelingen. Vielmehr bleibt er an der Oberfläche der Phänomene, die er in ihrer facettenreichen Fülle als »observador ambulatorio« wahrnimmt (EBA, 155), das heißt als beobachtender Flaneur. Wenn er in Explicación de Buenos Aires die Straßen der argentinischen Metropole durchstreift, setzt er den Habitus seiner literarischen Promenaden durch Madrid fort:

El madrileño es muy callejeador, y por eso yo puedo vivir en esta ciudad, en que la calle se abre en abanicos interminables. (EBA, 132)

Hier wird deutlich, dass Ramón nach der Absage an den Exotismus in Form und Inhalt auf das absolut Vertraute zurückgreift, um in der südlichen Hemisphäre, in Buenos Aires, seinen gewohnten Madrileñismo zu praktizieren. Der müßige Spaziergänger richtet seine Aufmerksamkeit auf die Signifikanz des Alltäglichen. Unter dem Titel »Andando y observando« (EBA, 30-33) beobachtet er beispielsweise die Arbeit der Schuhputzer oder hausierende Kinder, die Haarnetze verkaufen, bemerkt den Luxus der Juweliere, registriert absonderliche Details:

\footnotetext{
Existen los micromnibus, que parece que tienen por misión llevar a los microhombres.

Lo servicial está siempre pronto: «Si su piano está desafinado, llame al 354652». Bello título, leído al pasar: «Al chorizo honrado». [...]

Aquí se hacen los ensayos más originales de convivencia, y el Automóvil Club argentino ha ensayado una invitación de «¿Sube usted?», para peatones cansados de esperar tranvías. (EBA, 33)
}

Amüsierte Beobachtungen widmet er den »Pequeños negocios« (EBA, 3339), welche die Einwanderer im Kampf ums Überleben erfinden: etwa den Heimservice für Regenschirm-Reparaturen, die Vitamin-Bar oder die Glückwunsch-Agentur - »agencia de enhorabuenas« -, die gewiss eine Marktlücke füllt. Den ernsten sozialgeschichtlichen Hintergrund dieser 
Phänomene, dass nämlich der Mythos der Neuen Welt auch in ökonomischer Hinsicht ausgedient hat, erwähnt Ramón freilich nur beiläufig und auf humoristische Weise:

El hacer la América es cada vez más trabajoso, porque como oí el otro día a un porteño contestar a uno que decía, a grito pelado, que él había venido a «hacer la América):

- Caballero, la América ya está hecha... Llega usted tarde. (EBA, 36)

Der Zauber von El Dorado ist verflogen, der Optimismus Amerikas ${ }^{11}$ wird unterminiert von der Depression, die Ramón in Gestalt der »Tía Apa « thematisiert (EBA, 120-122). Das Augenmerk des spanischen Autors gilt ebenso den linguistischen Eigenheiten des Rio de la Plata ${ }^{12}$ wie auch den Besonderheiten des literarischen Lebens: Man schätzt illustrierte Gedichtbände und Vernissagen in angestaubter Atmosphäre (EBA, 167-169); auch Lyrik-Lesungen (EBA, 21-24) und Vorträge erfreuen sich großer Beliebtheit:

Sabido es que la Argentina es la primera consumidora de conferenciantes del mundo. (EBA, 69-72)

Unter den gastronomischen Spezialitäten erwähnt er die spezielle Vorliebe für den Aperitif, die sogenannten »copetines« (EBA, 48). Unerschöpfliches Amüsement bezieht der Flaneur schließlich aus den »chapas de portal« (EBA, 84-87), Namensschildern mit kuriosen Berufsbezeichnungen:

Una de las características de Buenos Aires son sus innumerables chapas de portal, en el marco de entrada, unas debajo de otras en lustrado bronce, algunas con antigüedad de epitafio: «Sociedad Wagneriana», "Conservatorio de Santa Cecilia», «Amigos del Dante».

Desde hace años sigo con curiosidad la estadistica de estas placas con sus nombres y sus apellidos desconcertantes, añadidas las especialidades y las caprichosas industriosidades que se apuntan en muchas de ellas. (EBA, 84)

Entre las cosas curiosas que se anuncian como repiquete he encontrado alguna como éstas: «Rejuvenecedor de pieles», «Detective de los cementerios», «Jurado de conejos») [...]. (EBA, 85)

11 Gelegentlich wird dieser Optimismus noch evoziert (EBA, 48 und 158).

12 Er erwähnt beispielsweise den "seseo« (EBA, 12), die Neigung zu Euphemismen (EBA, 63) und widmet dem häufig verwendeten Begriff „Chafalonia« (EBA, 165-167) sowie bestimmten »Frases y palabras diferenciales« (EBA, 94-97) wie "No más« und »iVelay! « ein eigenes Kapitel. 
Ein Flaneur, der auf sich hält, verachtet auch die Friedhöfe nicht. Sie zeugen vom verflossenen Mythos Amerikas, denn dort ruhen all jene "pioneros de la muerte en el Nuevo Mundo« (EBA, 56), deren Ziel es war, »[de] hacer la América« (EBA, 178). In den vielsprachigen Grabsteinen der Chacarita erblickt Ramón ein Abbild der kosmopolitischen Metropole:

El exotismo y extranjerismo de los nombres explica lo que es la nueva matriz de la urbe. $(\mathrm{EBA}, 178)$

In den Wohnvierteln der Lebenden gibt sich die Stadt noch deutlicher als internationaler Schmelztiegel zu erkennen. Es herrscht ein babylonisches Sprachengewirr, und die Straßen sind nach sämtlichen Regionen, Städten und Genies dieser Welt benannt. Buenos Aires fungiert insofern als multikultureller Mikrokosmos, als »universalidad sintetizada« (EBA, 133). Innerhalb dieses abrégé de l'univers ${ }^{13}$ nimmt Spanien einen privilegierten Platz ein, so dass die Straßen von Buenos Aires für Ramón zu einem >Echo<, einem >Spiegel< der Heimat werden: ${ }^{14}$

Todo este relato debe explicar cómo un nostálgico de España puede transitar con afición por este mundo, enrevesando en novelería su españolismo, sorprendido por el incesante hallazgo, pertrechándose de rincones y recovecos y apuntando direcciones y tiendas para más detalladas excursiones en futuros días. (EBA, 134)

Die Passage ist aufschlussreich, stellt sich das Verhältnis zwischen Alter und Neuer Welt hier doch so dar, dass Buenos Aires dem spanischen Fundus lediglich einen neuen Firnis verleiht. In der Tat kommt der südlichen Metropole kaum ein eigener Wirklichkeitsstatus zu, denn der Deutungshorizont aller Wahrnehmung und Erkenntnis bleibt letztlich Madrid. Der Blick des heimwehkranken Emigranten richtet sich auf das Gleiche im Anderen, die Alterität schwindet angesichts der hispanischen Affinität, und die Dialektik zwischen Fremdem und Eigenem verlagert sich zugunsten des Bekannten und Vertrauten:

Todo se explica, por España y por lo español, en el ancho estadio de la ciudad inmensa, y el andariego de calles y plazuelas es como sonámbulo de Madrid, que sigue vías paralelas en una especie de sueño real, en que la plaza de Mayo es co-

\footnotetext{
13 Cf. Angelika Corbineau-Hoffmann (1991).

14 Cf. EBA, 133: „En seguida pisamos aceras paralelas a las españolas, y es grato ser testigos de un eco inmenso, viendo en el gran espejo la grandeza lejana.«
} 
mo la Puerta del Sol y la avenida de Mayo es como la calle de Alcalá, llena de bazares, loterías, cafés con terraza con muchos limpiabotas [...]. (EBA, 134-135)

Hatte sich im Prosagedicht des Avantgardisten die antizipierende Imagination eines erträumten Amerika manifestiert, so tritt nun an deren Stelle der somnambule Tagtraum, der die wahrgenommene Realität reflexartig einer Metamorphose unterwirft, um immer wieder die rückblickende Vision eines erinnerten und ersehnten Madrid heraufzubeschwören. Die argentinisch-amerikanische Wirklichkeit gerät somit in den Verweisungszusammenhang eines sparallelen Universums`, wodurch sie nicht mehr in ihrer Singularität, sondern als bloße Reminiszenz und Spiegelung des heimatlichen Spanien wahrgenommen wird. Dieser Mikrostruktur entspricht das Spätwerk Ramóns auch in seiner Makrostruktur, bildet doch Explicación de Buenos Aires (1948) zusammen mit dem später entstandenen Band Nostalgias de Madrid (1956) gewissermaßen ein Diptychon. Die Topographie von Buenos Aires setzt sich zusammen aus lauter spanischen Sehnsuchtsorten. Die Avenida de Mayo evoziert auf diese Weise die »rambla catalana«, die Calle de Alcalá und die Gran Vía, so dass die Spanier sich dort zuhause fühlen und promenieren »como Pedro por su casa« (EBA, 183-184). Bereits im einleitenden Kapitel »Buenos Aires es...« wird die Kapitale des Cono Sur ausschließlich über Spanien definiert:

Buenos Aires es...

[...] Preguntarse: "¿Qué hora será allá?», y hacer ese cálculo que tiene algo de prestidigitación, adivinación del pensamiento y doblaje del tiempo.

Encontrar una lata de aceite titulada «Personajes», y ver que en ella está reproducido a todo color «El caballero de la mano en el pecho».

Llegar a una Sevilla que estuviese en Cataluña [...].

Ver cómo se repite el mundo, aun habiendo venido a parar tan lejos [...]. (EBA, 9)

So sehr Ramón Argentinien schätzt ${ }^{15}$, so verstörend wirkt doch gelegentlich der Unterschied zwischen >acá «nd >allá<: ein anderer Himmel ${ }^{16}$, ein anderes Klima, so dass Weihnachten im Hochsommer gefeiert ${ }^{17}$ und der

15 Cf. NPV, 173-174.

16 Cf. in dem Kapitel »El cielo de aquí y a Cruz del Sur « (EBA, 13): »Lo que más nos desconcierta precisamente es que este cielo sea diferente al otro, al que hemos tenido sobre nuestra cabeza toda la vida.«

17 Cf. in dem Kapitel »Verano de aquí (EBA, 63): »Lo que nos sorprende siempre al estar aquí es el que las del Año Nuevo sean fiestas bajo el imperio del sudor." 
Kunstgenuss des traditionellerweise an Allerheiligen aufgeführten Don Juan nachhaltig beeinträchtigt wird:

He visto aquí muy buenos Don Juanes [...]; pero en la tertulia del bodegón y de la gran cena había como una animación de Nochebuena pasada lejos del sitio indicado. - ¡Cuántas millas hasta Sevilla!

Todo en la representación del Don Juan aquí tiene un punto de añoranza, de melancolía lejana, de buñuelos de viento que se sabe que se están comiendo en otro lado.

$[\ldots]$ se sale del teatro con un vacío en el corazón [...]. (EBA, 175-176)

Da die Wahrnehmung von Differenz schmerzhaft ist, werden die Unterschiede eskamotiert und zu einer >Wiederkehr des Gleichen (nivelliert »Ver cómo se repite el mundo, aun habiendo venido a parar tan lejos« (EBA, 9). Dem von Verlusterfahrungen traumatisierten Emigranten ist es ein täglicher Trost, im Fremden das Eigene zu entdecken. Das beginnt bei recht banalen Dingen: Auch in Buenos Aires findet Ramón »Chorizo de Cantimpalos« (EBA, 98-102) und kann seine Gäste mit »Manzanilla« und »Mantecados« bewirten, ein Akt hispanischer Kommunion, der mehr bewirke als jeder »discurso de unión iberoamericana«:

Yo me quedo satisfecho, como si hubiese dado comunión española - tierra y vino espiritual -, a los que sólo adivinan vagamente lo que es nuestra España. (EBA, 88)

Der Klang von Kastagnetten schließlich, Herzschlag Spaniens - »ese latir de España« (EBA, 91) -, bedeutet ihm Lebensimpuls und Wiederauferstehung der Heimat in der Ferne. ${ }^{18}$

Offensichtlich handelt es sich bei dieser selektiven Wahrnehmung weniger um eine ideologisch motivierte >Hispanisierung Lateinamerikas als vielmehr um eine individualpsychologische Kompensationsstrategie. Dennoch verweist auch diese auf herrschende Hispanitäts-Diskurse und Ideologeme wie sie in Ortega y Gassets Argentinien-Essays ${ }^{19}$ oder der Polemik

18 Cf. das Kapitel »Las castañuelas que pasaron el mar« (EBA, 91-93). Die Wiederauferstehung der Kastagnetten in der Ferne - "su resurrección en la lejania« (EBA, 92) - verleiht ihnen eine tiefere Bedeutung: »Tocadas por ese mundo de castizas bailadoras que llenan la noche porteña, tienen un retruco de triunfo de la madre patria, en sus otras playas, que son el sacar la cabeza después de interminable nado desde la otra orilla. [...] Duchados de castañuelas, avivados por ellas, agilizados por su fosforescencia, encontramos en la ciudad americana un estímulo más, para seguir viviendo [...].«(EBA, 93)

19 Ein kontrastiver Vergleich zwischen Ramóns Argentinien-Erfahrung und Ortegas Essays »Meditación del pueblo joven«, "Meditación de la criolla« usw. wäre gewiss aufschlussreich. 
um Madrid als »meridiano intelectual de Hispano-América ${ }^{20}$ entworfen werden. Neben rein materiellen »símbolos españolistas« (EBA, 92) wie etwa den Kastagnetten oder dem »Chorizo« finden wir bei Ramón daher auch explizit hispanozentrische Konzepte wie das des »criollo«:

La Argentina tiene el criollo más claro, el que nos puede ser más idóneo, porque apenas tiene mestizaje, que es lo que más aparta del criollismo. (EBA, 25)

Aquí lo criollo es generalmente lo español, magnificado, con rumbo, fácil de interpretar con nobleza, con un fondo de castellanía vieja, más ablandada por el agua, con rotundidad magnificente y regalona. (EBA, 26)

In diesem Sinne begründet Ramón sein freiwilliges Exil in Argentinien auf dialektische Weise, insofern nämlich die zweite Heimat eine vertiefte Selbstvergewisserung der spanischen Identität erlaube, »porque pued[o] saborear mejor la esencia española« (NPV, 172-173). Die Emigration bedeutet für Ramón eine traumatische Dezentrierung, die er nur durch das weitgehende Eskamotieren von Alterität zu kompensieren vermag. Die radikale Bedeutung solcher Erfahrungen von kultureller Horizontverschiebung unterstreicht Roland Barthes:

C'est la dépossession de l'Occident qu'elle implique, l'image nouvelle qu'elle impose: celle d'un champ dont le sujet occidental n'est plus le centre ou le point de vue. ${ }^{21}$

Der vielbeschworene Wechsel der Perspektive, das heißt des point de vue bzw. des »balcón« mag Ramón nicht gelingen. Indem er sich darauf kapriziert, die Realität der Neuen Welt in den Kategorien der Alten wahrzunehmen, versucht er sich als »sujet occidental« zu behaupten. Doch die tiefgreifende Wirkung der »dépossession de l'Occident« schlägt sich in eindrücklichen Bildern nieder, welche das Gefühl der Heimatlosigkeit in Begriffe zu fassen versuchen. Das Bild der Arche Noah, das bereits in Explicación de Buenos Aires in Erscheinung tritt ${ }^{22}$, verdichtet sich in Nuevas páginas de mi vida zur Metapher einer existentiellen >Unbehaustheit<, die nur noch das Überleben angesichts einer kosmischen Katastrophe kennt:

20 Cf. den entsprechenden Artikel in La Gaceta literaria 8 (April 1927).

21 Roland Barthes, »Le refus d'hériter«, zitiert nach Ottmar Ette (1994: 297).

22 So vergleicht er die Avenida de Mayo mit der Arche Noah (EBA, 185), und im Gespräch mit dem ebenfalls emigrierten Pitigrilli heißt es: »Este es el monte Ararat, donde aterrizó el Arca de Noé después del diluvio - me dice con tono bíblico.« (EBA, 164) 
Estar aquí es tener opción a un puesto en el Arca de Noé - del Noé futuro - navegando bajo la lluvia del próximo Diluvio Universal. (NPV, 173)

In Anbetracht eines bevorstehenden Kataklysmus, der von der privaten in eine universelle Dimension überhöht wird, gerät Buenos Aires zum Refugium inmitten des Nichts, »el escondrijo en que me he retirado de ambos mundos« (NPV, 173). Jenseits von Alter und Neuer Welt befindet sich Ramón im argentinischen Exil an einem utopischen Ort. Diesem räumlichen Nirgendwo entspricht die Vorstellung, ein `Endzeitler $<$ zu sein - »soy un finalista del mundo « (NPV, 172) -, der isoliert in einer verfinsterten Welt lebt, »en un mundo ensombrecido y sin correspondencia ${ }^{23}$. Ohne jeglichen Kontakt zu seinen argentinischen Schriftstellerkollegen ${ }^{24}$ zieht sich Ramón immer mehr in das Madrid seiner Erinnerung zurück und vermag die Horizontverschiebung daher nicht als Horizonterweiterung und Erkenntnisgewinn zu nutzen:

Aquí estamos un poco ennichados en nuestro laboreo, nichal alegre y cómodo desde el que asistimos silenciosa y escondidamente al sobrepasarse de esta gran cosmopolitanía del Sur. (EBA, 118)

Kehren wir abschließend nochmals zurück auf die imaginäre Vorwegnahme Amerikas in den »Nouveaux paysages imaginaires« des Jahres 1923. Ramón hatte sich damals ein amerikanisches alter ego erdacht, das ihm jenseits des Ozeans spiegelgleich gegenüber sitzt:

Il est assis à sa table aux mêmes heures que moi, dans ce coin d'Amérique, et il écrit les mêmes choses. Je sais qu'il lui est plus difficile qu'à moi de les éditer, que ce lui est même presque impossible, mais il les écrit. [...]

C'est un type comme moi, [...], avec le même regard perdu...

Seul, le paysage de la fenêtre est différent. Le sien est un paysage de vaste perspective, un paysage qui galope et où se voit courir la plate-forme roulante du monde. (NPA, 193)

Durch das Exil nimmt Ramón den Platz des imaginierten Anderen ein. Doch statt dessen »vaste perspective « zu gewinnen ${ }^{25}$, kehrt er den spiegelbildlichen Blick einfach um. Er blickt zurück auf sein altes Ich in Madrid

23 Ramón Gómez de la Serna (1962: 97).

24 Cf. Kapitel wie "La vida literaria« (EBA, 105-108), "No nos vemos« (EBA, 118-120) oder »Camafeo de la soledad « (NPV, 84-90).

25 Cf. EBA, 188: "La ancha terraza desde la que se domina el poliorama de los sucesos, concede un punto de vista de lo más imparcial y anchuroso. Sólo aqui se produce esa prodigiosa supervisión de la realidad. « 
und treibt angesichts der Vereinsamung des Exils die Persönlichkeitsspaltung so weit, dass er Briefe an sich selbst schreibt, Cartas a mí mismo. ${ }^{26} \mathrm{Im}$ Vorwort erläutert er:

La gran soledad del presente me hizo comenzar en América esta correspondencia conmigo mismo, $[\ldots]$ al escribirse uno a sí mismo se escribe a un hombre más joven que uno mismo. (CMM, 97)

Indem er den Stil dieser Briefe charakterisiert, kennzeichnet er einmal mehr seine Lebenssituation in Buenos Aires; er schreibe nämlich »desgarradoramente, con esta sinceridad desplanchada, estilo fosa común, estilo emigrante sin nadie en el mundo« (CMM, 98). Ramón fokussiert seinen Blickwinkel immer stärker auf Spanien. ${ }^{27}$ Das imaginierte Amerika des Avantgardisten wird vom erinnerten Spanien des Emigranten abgelöst, während das erlebte Buenos Aires nur durch eine sspanische Brille wird. ${ }^{28}$ Einige Aspekte dieser interkulturellen Erfahrung und ihrer Dialektik haben wir kurz beleuchtet. Zwischen den beiden Vaterländern ${ }^{29}-$ dem spanischen und dem hispanisch-kreolisch-kosmopolitischen - vollzieht sich für das Subjekt, das an beiden partizipiert, gewiss ein wechselseitig erhellender Prozess von Alteritätswahrnehmung und Selbstreflexion, allerdings keineswegs in jener idealen Komplementarität, wie sie Ramón zu Beginn seiner Explicación de Buenos Aires postuliert:

Caminamos así por veredas de España sobre la gran extensión del pueblo nuevo, que tiene una inmensa alma propia, y pensamos que igual le debe suceder al argentino que va de aquí a allí cuando se siente caminar por sendas argentinas entreveradas en el mapa español.

Por esas venas invisibles [...] vivimos en la circulación de la patria lejana. (EBA, 12)

26 In Clavileño veröffentlicht Ramón im Jahre 1953 »Paquete de cartas a mí mismo« und »Nuevo paquete de cartas a mí mismo«, gefolgt von einem »Último paquete de cartas a mí mismo« (1954); die gesammelten Briefe erscheinen im Jahre 1956 unter dem Titel Cartas a mi mismo.

27 Hier lässt sich anknüpfen an die im Beitrag von Vittoria Borsò erörterte Problematik des Blicks.

28 Bereits anlässlich seines ersten Besuchs in Buenos Aires charakterisiert Victoria Ocampo (1931) ihn als »violentamente, agresivamente español«.

29 Cf. NPV, 173: "Esta no es una segunda patria, sino una patria equivalente en la que al mismo tiempo estamos de incógnito." 
Imaginiertes Amerika, erinnertes Spanien, erlebtes Argentinien

\section{Bibliographie}

Literarische Werke und andere Quellen

[La] Gaceta literaria 8 (April 1927).

Gómez de la Serna, Ramón (1922): »Paysages imaginaires d'Amériques«, Revue de l'Amérique Latine I: 4, pp. 314-320.

Gómez de la Serna, Ramón (1923): »Nouveaux paysages imaginaires d'Amérique«, Revue de l'Amérique Latine IV: 3, pp. 193-199.

Gómez de la Serna, Ramón (1975 [1948]): Explicación de Buenos Aires, Buenos Aires: Ediciones de La Flor.

Gómez de la Serna, Ramón (1962 [1956]): Cartas a las golondrinas. Cartas a mi mismo, Madrid: Espasa-Calpe.

Gómez de la Serna, Ramón (1970 [1957]): Nuevas páginas de mi vida: lo que no dije en mi Automoribundia, Madrid: Alianza.

Gómez de la Serna, Ramón (1999): La ciudad. Madrid, Buenos Aires (1919-1956), hrsg. v. Iona Zlotescu, Barcelona: Galaxia Gutenberg.

Ramón en cuatro entregas (1980), hrsg. v. Juan Manuel Bonet, Madrid: Museo Municipal.

Forschungsliteratur

Albert, Mechthild (1991): »Ramón Gómez de la Serna und die lateinamerikanische Avantgarde - Vom Mythos zum Dialog«, in: Wentzlaff-Eggebert, Harald (Hrsg.): Europäische Avantgarde im lateinamerikanischen Kontext, Frankfurt am Main: Vervuert, pp. 147-162.

Castro, Donald S. (1995): »The Gaucho Revival in Argentina in the 1930s and 1940s: The Gaucho as a Symbol of National and Social Values in the Works of Elbio Bernardez Jacques«, Studies in Latin American Popular Culture 14, pp. 171-198.

Corbineau-Hoffmann, Angelika (1991): Brennpunkt der Welt. "C'est l'abrégé de l'univers«. Großstadterfahrung und Wissensdiskurs in der pragmatischen Parisliteratur 1780-1830, Bielefeld: Schmidt.

Ette, Ottmar (1994): »Asymmetrie der Beziehungen. Zehn Thesen zum Dialog der Literaturen Lateinamerikas und Europas«, in: Scharlau, Birgit (Hrsg.): Lateinamerika denken. Kulturtheoretische Grenzgänge zwischen Moderne und Postmoderne, Tübingen: Narr, pp. 297-326.

Mahler, Andreas (1999): »Stadttexte - Textstädte. Formen und Funktionen diskursiver Stadtkonstitution«, in: id. (Hrsg.): Stadt-Bilder. Allegorie, Mimesis, Imagination, Heidelberg: Winter, pp. 11-36.

Ocampo, Victoria (1931): »Ramón Gómez de la Serna en Buenos Aires«, Sur I: 2, pp. 205-208.

Paris et le phénomène des capitales littéraires. Carrefour ou dialogue des cultures (1984). Actes du premier congrès international du CRLC, 4 Bde., Paris: Presses de l'Université de Paris-Sorbonne.

Rössner, Michael (1988): Auf der Suche nach dem verlorenen Paradies. Zum mythischen Bewußtsein in der Literatur des 20. Jahrhunderts, Frankfurt am Main: Athenäum. 